\title{
Importance d'optimiser l'afférence visuelle chez la personne âgée vulnérable vivant en institution
}

\section{PAR ESTEFANIA CHRIQUI OD, MSC (CANDIDATE) ${ }^{1,2}$ \\ NATHALIE CHAMPOUX MD, MS ${ }^{2,3}$ HÉLÈNE KERGOAT OD, PhD ${ }^{1,2}$}

E n $2009,14 \%$ de la population canadienne était âgée de 65 ans ou plus, une proportion qui s'élèvera à environ $24 \%$ en $2036 .{ }^{1}$ En 2008-2009, plus de 155000 personnes âgées de plus de 65 ans au Canada vivaient en institutions de soins de longue durée, dont environ 40000 au Québec. ${ }^{2} \mathrm{Au}$ cours des dernières années, il y a eu réorganisation des services de santé, avec un mouvement vers la communauté, incluant entre autres une augmentation des services ambulatoires et des soins à domicile. ${ }^{3}$ Un effet lié à ces changements est que les personnes âgées admises en soins de longue durée sont de plus en plus dépendantes, ont des maladies plus complexes, et davantage de désordres moteurs et sensoriels. ${ }^{4}$ Entre 60 et $80 \%$ d'entre elles présentent un déficit d'ordre cognitif, ${ }^{4}$ ce qui n'est pas surprenant sachant qu'au-delà de $8 \%$ de la population canadienne âgée de 65 ans et plus est atteinte de la maladie d'Alzheimer ou d'autres formes de démence. ${ }^{5}$ Cette prévalence dépasse les 30\% chez les gens âgés de 85 ans ou plus. ${ }^{5}$ Le vieillissement de la population canadienne, avec l'augmentation de prévalence de la démence et la complexité des maladies au grand âge, mettra au défi le système de santé et entraînera une nécessaire adaptation de l'offre des soins et services.

Il est préférable que les personnes âgées puissent demeurer chez elles le plus longtemps pos-

\section{RÉSUMÉ}

L'acuité visuelle est une mesure importante effectuée lors de l'examen visuel afin de vérifier la capacité d'une personne à bien voir et guider l'ajustement de la réfraction oculaire. II s'agit d'un test de routine qui s'effectue généralement en quelques minutes et sans difficulté particulière. Cette mesure peut par contre être plus difficile à obtenir chez la personne âgée atteinte de troubles de la cognition ou de la communication. Ceci pourrait être le cas par exemple chez la personne âgée atteinte de démence ou celle souffrant d'aphasie suite à un accident vasculaire cérébral. Le but de cet article est de présenter certains aspects du vieillissement de la population, des données liées à l'institutionnalisation de la personne âgée, diverses conditions plus prévalentes liées à l'âge, ainsi que des facteurs particuliers démontrant l'importance d'optimiser la vision chez les personnes âgées. Finalement, cet article fera un survol de diverses échelles disponibles pour mesurer l'acuité visuelle.

Mots clefs : Vision, personne âgée vulnérable, institutionnalisation, acuité visuelle

1 École d'optométrie, Université de Montréal

2 Institut universitaire de gériatrie de Montréal

3 Faculté de médecine, Université de Montréal

sible afin de maintenir l'intégrité de leur réseau social, de préserver leurs repères environnementaux, et de pouvoir ainsi profiter d'une meilleure qualité de vie. Malgré cela, la complexité des maladies, la sévérité des incapacités en découlant, ainsi que la présence de problèmes comportementaux sont autant de facteurs qui conduiront tôt ou tard à leur entrée en institution de soins de longue durée. ${ }^{6}$ L'institutionnalisation est souvent davantage liée à la sévérité des incapacités découlant de la maladie qu'à la maladie elle-même. Certaines caractéristiques liées à l'aidant naturel, comme un âge plus avancé, l'épuisement ou la dépression, peuvent aussi conduire à une institutionnalisation plus hâtive de la personne âgée dont on prend soin. ${ }^{6}$

Au Québec, environ 4,7\% de la population âgée de 65 ans ou plus réside en Centre d'hébergement et de soins de longue durée (CHSLD). Ce milieu de vie est réservé aux adultes en perte d'autonomie fonctionnelle ou psychosociale qui requièrent trois heures ou plus de soins pour les aider dans leurs activités de la 
vie quotidienne. Il s'agit donc de personnes « vulnérables » qui ont besoin d'aide et de soins appropriés pour fonctionner au quotidien. La notion de vulnérabilité chez la personne âgée évolue constamment, mais peut entre autres se définir ainsi «des personnes généralement âgées de plus de 75 ans, qui en raison d'une accumulation de multiples affections chroniques, nécessitent souvent un ou plusieurs services de soutien afin de faire face aux activités de la vie quotidienne ».7 La vulnérabilité peut donc être considérée comme une fragilité croissante liée au vieillissement. ${ }^{8}$ Outre les problèmes de démence mentionnés auparavant, d'autres maladies entraînant des incapacités sévères peuvent aussi donner lieu au placement de la personne âgée en CHSLD, comme les troubles de l'humeur, le syndrome parkinsonien, l'accident vasculaire cérébral, les maladies vasculaires ou pulmonaires chroniques. La durée moyenne de séjour en CHSLD peut aller jusqu'à 3 ans ${ }^{9}$ et constitue souvent le dernier milieu de vie de la personne âgée jusqu'à son décès. Il est donc important d'optimiser les soins et la qualité de vie dans le respect des volontés du patient, exprimées par ce dernier, ou par la personne répondant pour le patient, puisqu'une grande majorité de ces patients ne sont plus aptes à prendre des décisions éclairées quant à leur santé.

Plusieurs maladies affectant la personne âgée peuvent entraîner des troubles de la communication et rendre les examens et interven- tions visuelles plus difficiles. C'est le cas par exemple pour les troubles sévères de la cognition dans la démence, l'aphasie liée à l'accident vasculaire cérébral, la lenteur/difficulté d'élocution accompagnant parfois la maladie de Parkinson, l'humeur apathique du patient atteint de dépression majeure, les craintes souvent répétées de la personne atteinte de troubles anxieux, la surdité etc. Malgré les difficultés, l'évaluation visuelle est importante car la déficience visuelle est prévalente au sein de la population âgée. À l'échelle mondiale, 285 millions de personnes présentent une déficience visuelle et près de $65 \%$ de l'ensemble de ces personnes sont âgées de 50 ans et plus. ${ }^{10} \mathrm{Au}$ Canada, les données indiquent que 278000 canadiens sont atteints d'une déficience visuelle, que 108000 sont légalement aveugles et qu'un canadien sur trois âgé de plus de 75 ans éprouverait un certain niveau d'incapacité visuelle. ${ }^{11}$ Par ailleurs, les études indiquent que la déficience visuelle est particulièrement élevée chez les personnes vivant en institution. ${ }^{12,13}$ De plus, la prévalence de la déficience visuelle et de la cécité augmente avec l'âge, quelle que soit l'appartenance ethnique, ${ }^{14-16}$ à cause principalement de la dégénérescence maculaire liée à l'âge, du glaucome ou de la cataracte. ${ }^{14,17}$ La perte visuelle peut aggraver les problèmes associés à la démence $^{18}$ et elle constitue un facteur indépendant contribuant aux troubles du comportement parmi les résidents vivant en institu- tion de soins de longue durée. ${ }^{19}$ Une grande partie des déficits visuels chez ces personnes sont dus à des conditions pouvant être traitées comme les erreurs de réfraction non corrigées ou les cataractes. ${ }^{12,20}$ Ces conditions doivent être dépistées et traitées lorsque possible. Ces constats sont d'autant plus alarmants sachant que les personnes âgées vivant en institution ne reçoivent pas nécessairement des services oculovisuels adéquats, ${ }^{21}$ et que la correction de la réfraction et la chirurgie de la cataracte peuvent améliorer considérablement leur qualité de vie tout en diminuant leurs symptômes de dépression. ${ }^{22-24}$

La pertinence d'effectuer une chirurgie de cataracte chez la personne âgée vivant dans la communauté est bien documentée. Les bénéfices de la chirurgie de la cataracte chez les personnes âgées vivant en institution et n'ayant pas de troubles cognitifs importants ont aussi été démontrés. ${ }^{24}$ Malgré cela, la chirurgie de la cataracte n'est pas nécessairement effectuée de routine chez les personnes âgées institutionnalisées. Une étude indique effectivement que la chirurgie de la cataracte est moins bien acceptée chez les gens vivant en soins de longue durée. Cette étude rapporte que les personnes âgées ayant un déficit cognitif sont moins à même de prévenir leur entourage de leurs problèmes visuels, que leurs aidants naturels sont plus réfractaires aux procédures chirurgicales, que les bénéfices de la chirurgie peuvent sembler moins 
évidents et qu'il est plus difficile de tester leur vision. ${ }^{25}$ L'évaluation subjective de l'acuité visuelle peut représenter un défi au sein d'une population institutionnalisée qui présente des troubles importants de la cognition ou de la communication, et le jugement clinique doit parfois être utilisé pour définir l'acuité visuelle approximative du patient. ${ }^{26}$ Malgré cela, une étude clinique rétrospective a démontré qu'il était possible de réaliser un examen visuel complet chez les personnes âgées vivant en institution, indépendamment de l'âge avancé, du statut cognitif et des troubles de la communication. ${ }^{17}$ De plus, l'acuité visuelle était préservée à un âge avancé dans l'œil non atteint de pathologie oculaire. ${ }^{17}$ Cette étude suggère donc qu'il est possible d'évaluer ces personnes âgées, mais que l'approche clinique doit être adaptée afin de maximiser l'information obtenue dans le cadre de l'examen visuel.

Une autre problématique ciblant particulièrement la personne âgée et où la vision peut jouer un rôle important est la chute. ${ }^{27}$ Les chutes constituent effectivement un problème de santé majeur sachant que $30 \%$ des personnes âgées de 65 ans et plus font au moins une chute par année, ${ }^{28}$ que cette proportion atteint $50 \%$ chez les plus de 85 ans vivant à la maison ${ }^{29}$ et qu'elle est encore plus élevée chez les personnes âgées vivant en institution. ${ }^{30}$ De plus, $5 \%$ des personnes âgées qui chutent doivent être hospitalisées. ${ }^{31} \mathrm{Au}$
Québec, les chutes donnant lieu à un traumatisme représentent 10 à 15\% des admissions en unités de courte durée gériatrique. ${ }^{32}$ Les chutes sont d'origine multifactorielle. ${ }^{33} \mathrm{La}$ déficience visuelle a maintes fois été citée comme étant un facteur de risque de chute et de fracture chez la personne âgée. ${ }^{34}$ Plusieurs études, entre autres, ont démontré qu'une diminution d'acuité visuelle représentait un risque indépendant de chute chez la personne âgée, ${ }^{34-36}$ même si cela n'est pas universellement accepté. ${ }^{37,38}$ L'évaluation de la vision chez la personne âgée ayant chuté est donc importante et devrait être systématique, ${ }^{33}$ bien que ce ne soit pas le cas actuellement. ${ }^{39}$ Les études indiquent, entre autres, que la diminution d'acuité visuelle, la perte de sensibilité aux contrastes, la diminution de vision stéréoscopique et la perte de champ visuel peuvent augmenter le risque de chute chez la personne âgée. .0-43 $^{-4}$ La chirurgie de la cataracte diminue les risques de chute et de fracture ${ }^{44}$ de même que les blessures. ${ }^{45}$ Un essai clinique randomisé a également démontré que la chirurgie de la cataracte du premier œil chez la femme âgée diminuait les risques de chute et de fracture, de même que l'anxiété et la dépression, tout en améliorant l'acuité visuelle et la sensibilité aux contrastes. ${ }^{46} \mathrm{La}$ chirurgie de cataracte du deuxième œil au sein de cette même population était associée à une amélioration de la fonction visuelle ainsi qu'à une diminution de la crainte de chuter et du niveau de handicap. ${ }^{47}$ Malgré les résultats obtenus dans ces études, il n'y a pas encore suffisamment d'évidence pour indiquer de façon non équivoque que la chirurgie de la cataracte diminue les chutes chez les personnes âgées. ${ }^{48} \mathrm{Il}$ demeure toutefois important d'évaluer la vision chez la personne âgée ayant chuté ou à risque de chute, et de l'optimiser chaque fois que cela est possible, que ce soit par traitement optique, médical, chirurgical ou par réadaptation visuelle. ${ }^{49}$

L'acuité visuelle correspond à la capacité de résolution spatiale de l'œil et se mesure cliniquement en identifiant l'angle sous-tendu à l'œil par le plus petit optotype reconnaissable. L'outil standard de prise d'acuité visuelle au sein de la population générale demeure l'échelle de Snellen traditionnelle, constituée de lettres ou optotypes à contraste élevé diminuant en grandeur de haut en bas de l'échelle. ${ }^{50}$ En présence de personnes âgées ayant des troubles importants de la cognition, altérant la compréhension de consignes et la communication, il n'est pas toujours possible de mesurer l'acuité visuelle avec l'échelle de Snellen. Il en est de même pour d'autres populations cliniques, et au fil des ans, de nouvelles échelles de mesure de l'acuité visuelle ont été élaborées pour répondre à des besoins particuliers. L'échelle logarithmique, dont la progression de la hauteur des lettres d'une ligne à l'autre est géométrique, ${ }^{51}$ offre une mesure plus standardisée de l'acuité visuelle et 
son utilisation est privilégiée dans l'évaluation des patients en basse vision ${ }^{52}$ et dans les études épidémiologiques. ${ }^{53}$ L'échelle des symboles de Lea a été conçue pour mesurer l'acuité visuelle chez les enfants de 3 à 5 ans, ${ }^{54}$ et structurée de telle sorte que l'enfant puisse répondre soit en pointant ou en nommant des symboles faciles à identifier à cet âge..$^{55}$ La méthode du regard préférentiel a été élaborée afin de tester des personnes atteintes de handicap intellectuel. ${ }^{56} \mathrm{Il}$ s'agit d'une technique comportementale basée sur le principe qu'en présence de deux cibles, l'une formée d'un réseau de lignes noires et blanches, l'autre formée d'une plage grise uniforme, une personne préfèrera regarder le réseau. ${ }^{57,58}$ La personne continuera à préférer le réseau, lors de la présentation de cibles successives comportant une plage grise et un réseau où la fréquence spatiale des lignes noires et blanches est augmentée, tant qu'elle sera en mesure de discriminer les lignes.

Aucune échelle d'acuité visuelle à ce jour n’a été développée spécifiquement pour la personne âgée atteinte de déficits sévères de la cognition ou de troubles importants de la communication. La méthode du regard préférentiel (cartes de Teller) aurait par contre été utilisée avec succès cliniquement chez un groupe restreint de personnes âgées en soins de longue durée ayant des problèmes de communication ${ }^{59}$ et des déficits cognitifs modérés. ${ }^{60}$ Par ailleurs, dans le cadre d'une étude effectuée en soins de longue durée, des chercheurs ont démontré que l'acuité visuelle pouvait être testée chez $84 \%$ des résidents à l'aide des cartes de Teller, contre $74 \%$ des participants lorsque l'échelle ETDRS ou Lea était utilisée. ${ }^{61} \mathrm{Il}$ est important de souligner, par contre, que cette étude incluait uniquement des gens ayant un déficit cognitif léger à modéré, les chercheurs ayant exclus d'emblée les résidents ayant un déficit cognitif plus sévère.

Les études présentées dans ce manuscrit démontrent qu'il est important d'évaluer la fonction visuelle $^{12,21}$ et la santé oculaire ${ }^{14,20}$ chez la personne âgée, d'autant plus que la pathologie oculaire et la perte visuelle présentent une prévalence accrue avec l'âge. De plus, une bonne afférence visuelle est un facteur jouant un rôle important dans la qualité de vie de la personne âgée $22-24$ alors que la perte visuelle peut contribuer à l'anxiété, ${ }^{62}$ à la dépression, ${ }^{63}$ à l'isolement, ${ }^{62}$ aux troubles de comportement ${ }^{19}$ et aux chutes. ${ }^{34,35}$ Ces phénomènes s'aggravent chez la personne âgée vivant en institution, ${ }^{12,13}$ car elles sont plus vulnérables du fait de leur état de santé fragile et de leur perte d'autonomie. ${ }^{6}$ Les troubles cognitifs qui affectent une large portion de ces personnes peuvent diminuer leur capacité à exprimer leurs besoins et leurs difficultés le cas échéant. ${ }^{25}$ L'évaluation de leur santé, incluant les soins oculovisuels, est souvent plus difficile, doit être adaptée pour les besoins spécifiques de cette population et demande un peu plus de temps. ${ }^{17}$ Ces personnes peuvent aussi avoir de la difficulté à communiquer et à collaborer lors de l'examen visuel, ce qui peut affecter une mesure aussi simple mais tellement importante que l'acuité visuelle. ${ }^{64}$

Il n'existe pas d'échelle « universelle » permettant de mesurer l'acuité visuelle chez les personnes âgées vulnérables ayant de la difficulté à communiquer ou à collaborer. Dans un cadre clinique, la prise d'acuité visuelle se déroule souvent en présentant les diverses échelles de mesure existantes une à la suite de l'autre, jusqu'à ce que l'une d'entre elles attire suffisamment l'attention du patient pour lui permettre de collaborer. Il n'existe aucune étude à ce jour pour guider le clinicien. Il serait donc important de savoir s'il existe une échelle supérieure aux autres pour mesurer adéquatement l'acuité visuelle chez les personnes âgées vulnérables, ou encore s'il est possible d'optimiser leur ordre de présentation, afin d'accélérer l'examen visuel tout en minimisant le degré de fatigue chez le patient. Une telle étude est actuellement en cours et les résultats feront l'objet d'une publication ultérieure. 


\section{Remerciements}

L'écriture de ce rapport a été réalisée grâce à une subvention du Fonds de fiducie des optométristes canadiens pour l'éducation à EC et $\mathrm{HK}$.

\section{Références}

1. Statistics Canada, 2010. Population and demography. Canada Year Book 2010. Catalogue no. 11-402-X. p. 315

2. Statistics Canada, 2011. Residential Care Facilities 2008/2009. Catalogue no. 83$237-X$. p. 50 , p. 55

3. Un milieu de vie de qualité pour les personnes hébergées en CHSLD. Orientations ministérielles. 2003. http://www.msss.gouv.qc.ca/sujets/ groupes/personnes_agees.php\#milieu Accès : 4 septembre 2011.

4. The Canadian Study of Health and Aging Working Group. Canadian Study of Health and Aging: study methods and prevalence of dementia. Can Med Assoc J. 1994; 150: 899-913.

5. The Canadian Study of Health and Aging. Canadian Study of Health and Aging: Methods and Prevalence of Dementia (incl. Kergoat M-J). Can Med Assoc J 1994; 150: 899-914.

6. Hébert R, Dubois M-F, Wolfson C et al. Factors associated with long-term institutionalization of older people with dementia: data from the Canadian study of health and aging. Journal of Gerontology: Medical Sciences 2001; 56A (11): 693-99.

7. Hogan DB, MacKnight C, Bergman H. Models, definitions, and criteria of frailty. Aging Clin Exp Res 2003;15:1-29.

8. Slaets JP. Vulnerability in the elderly: frailty. Med Clin North Am. 2006;90: 593-601.

9. Rapport annuel IUGM 2010-2011. http://www.iugm.qc.ca/iugm/ publication/publications-iugm/rapportsen-ligne

10. Organisation mondiale de la santé (OMS) : Cécité et déficience visuelle, Aide mémoire \#282, Octobre 2011. http://www.who.int/mediacentre/ factsheets/fs282/fr/
11. The National Coalition For Vision Health. Foundations for a Canadian Health Strategy. Towards Preventing Avoidable Blindness and Promoting Vision Health. Prepared for the National Coalition for Vision Health. January 2007.

12. Owsley C, McGwin G, Scilley K et al. The visual status of older persons residing in nursing homes. Arch Ophthalmol 2007; 125:925-930.

13. Jin YP, Wong DT. Self-reported visual impairment in elderly Canadians and its impact on healthy living. Can J Ophthalmol. Aug 2008; 43(4): 407-413.

14. The Eye Diseases Prevalence Research Group. Causes and Prevalence of visual impairment among adults in the United States. Arch Ophthalmol 2004; 122:47785

15. Yamada M, Hiratsuka $Y$, Roberts CB et al. Prevalence of visual impairment in the adult Japanese population by cause and severity and future projections. Ophthalmic Epidemiol. 2010 Jan-Feb; 17(1): 50-7.

16. Abdull MM, Sivasubramaniam S, Murthy GV et al. Causes of blindness and visual impairment in Nigeria: the Nigeria national blindness and visual impairment survey. Invest Ophthalmol Vis Sci. 2009 Sep; 50(9):4114-20.

17. Carcenac G, Hérard ME, Kergoat MJ et al. Assessment of visual function in institutionalized elderly patients. J Am Dir Assoc 2009; 10:45-49.

18. Coons D.H, Weaverdick S.E. Wesley hall: a residential unit for persons with Alzheimer's disease and related disorders. Physical and Occupational Therapy in Geriatrics 1986; 4:29-53

19. Horowitz A. The relationship between vision impairment and the assessment of disruptive behaviors among nursing home residents. The Gerontologist 1997; 37:620-28.

20. VanNewkirk MR, Weih LA, McCarthy $\mathrm{CA}$, et al. Cause-specific prevalence of bilateral visual impairment in Victoria, Australia. The visual impairment project. Ophthalmology 2001; 108:960-967.
21. Van der Pols J C, Bates C J, Mc Graw P $\mathrm{V}$, et al. Visual acuity measurements in a national sample of British elderly people. Br J Ophthalmol 2000; 84:165-170.

22. Coleman Al, Yu F, Keeler E, Mangione CM. Treatment of uncorrected refractive error improves vision-specific quality of life. J Am Geriatr Soc 2006 Jun; 54(6): 883-90.

23. Owsley C, McGwin G, Scilley K et al. Effect of refractive error correction on health-related quality of life and depression in older nursing home residents. Arch Ophthalmol 2007; 125:1471-1477.

24. Owsley C, McGwin G, Scilley K et al. Impact of cataract surgery on healthrelated quality of life in nursing home residents. Br J Ophthalmol 2007; 91:1359-1363.

25. Friedman DS, Munoz B, Bandeen Roche K, et al. Poor uptake of cataract surgery in nursing home residents. Arch Ophthalmol 2005; 123:1581-87.

26. VanNewkirk MR, Weih L, McCarthy CA, et al. Visual impairment and eye diseases in institutionalized Australians. Ophthalmology 2000; 107:2203-2208.

27. American Geriatrics Society, British Geriatrics Society, and American Academy of Orthopaedic Surgeons Panel on Falls Prevention. Guideline for the prevention of falls in older persons. J Am Geriatr Soc 2001; 49:664-672.

28. O'Loughlin JL, Robitaille Y, Boivin JF, et al. Incidence of and risk factors for falls and injurious falls among the communitydwelling elderly. Am J Epidemiol 1993; 137:342- 354 .

29. Formiga F, Ferrer A, Duaso E, et al. Falls in nonagenarians living in their own homes: The NonaSantfeliu study. J Nutr Health Aging 2008; 12:273-276

30. Masud T, Morris RO. Epidemiology of falls. Age and Ageing 2001; 30-S4: 3-7.

31. Bezon J, Echevarria KH, Smith GB. Nursing outcome indicator: Preventing falls for elderly people. Outcomes Manag Nurs Pract 1999; 3:112-116. 
32. Ministère de la santé et des services sociaux: Cadre normatif du système Med-Écho (Maintenance et exploitation des données pour l'étude de la clientèle hospitalière). Québec: Ministère de la santé et des services sociaux; 2009.

33. Summary of the Updated American Geriatrics Society/British Geriatrics Society Clinical Practice Guideline for Prevention of Falls in Older Persons. J Am Geriatr Soc 2011;59:148-157.

34. Harwood RH. Visual problems and falls. Age and Ageing 2001; 30-S4: 13-18.

35. Dargent-Molina P, Favier F, Grandjean H, et al. Fall-related factors and risk of hip fracture: the EPIDOS prospective study. Lancet 1996;348:145-149.

36. Ivers RQ, Norton R, Cumming RG, et al. Visual impairment and risk of hip fracture. Am J Epidemiol 2000;152:633639.

37. Freeman EE, Muñoz B, Rubin G, West SK. Visual field loss increases the risk of falls in older adults: The Salisbury eye evaluation. Invest Ophthal Vis Sci 2007;48:4445-4450.

38. Lord SR, Dayhew J. Visual risk factors for falls in older people. J Am Geriatr Soc. 2001;49:508-515.

39. Boutin T, Kergoat MJ, Latour J, Massoud F, Kergoat H. Vision in the global evaluation of older individuals hospitalized following a fall. J Am Dir Assoc. 2011. Sous presse.

40. Knudtson MD, Klein BEK, Klein R. Biomarkers of aging and falling: The BeaverDam eye study. Arch Gerontol Geriatr 2009; 49:22-26.

41. Lord SR, Clark RD, Webster IW. Physiological factors associated with falls in an elderly population. J Am Geriatr Soc 1991; 39:1194-1200.

42. Glynn RJ, Seddon JM, Krug Jr JH, et al. Falls in elderly patients with glaucoma. Arch Ophthalmol 1991; 109:205-210.

43. Jack CI, Smith T, Neoh C, Lye M, et al. Prevalence of low vision in elderly patients admitted to an acute geriatric unit in Liverpool: Elderly people who fall are more likely to have low vision. Gerontology 1995; 41:280-285.
44. Brannan S, Dewar C, Sen J, Clarke D, Marshall T, Murray PI. A prospective study of the rate of falls before and after cataract surgery. Br J Ophthalmol 2003;87:560-562.

45. De Coster C, Dik N, Bellan L. Health care utilization for injury in cataract surgery patients. Can J Ophthalmol 2007;42:567-572.

46. Harwood RH, Foss AJE, Osborn F et al. Falls and health status in elderly women following first eye cataract surgery: A randomized controlled trial. $\mathrm{Br} \mathrm{J}$ Ophthalmol 2005; 89:53-59.

47. Foss AJE, Harwood RH, Osborn F, Gregson RM, Zaman A, Masud T. Falls and health status in elderly women following second eye cataract surgery: A randomised controlled trial. Age Ageing 2006; 35:66-71.

48. Desapriya E, Subzwari S, Scime-Beltrano G, Samayawardhena LA, Pike I. Vision improvement and reduction in falls after expedited cataract surgery. Systematic review and metaanlysis. J Cataract Refract Surg. 2010;36:13-19.

49. Lord SR, Smith ST, Menant JC. Vision and falls in older people: Risk factors and intervention strategies. Clin Geriatr Med 2010;26;569-581.

50. Bennet AG. Ophthalmic test types. Br J Physiol Opt 1965; 22: 238-271.

51. Bailey IL, Lovie JE. New design principles for visual acuity letter charts. Am J Optom Physiol Opt 1976; 53: 740745.

52. Ferris FL, Kassof A, Bresnick GH, Bailey I. New visual acuity charts for clinical research. Am J Ophthalmol 1982; 94: 91 96.

53. Kassof A, Goodman D et al. Early treatment diabetic retinopathy study design and baseline patient characteristics. Ophthalmol 1991; 98: 741-751.

54. Hyvarinen L, Nasanen R, Laurinen P. New visual acuity test for pre-school children. Acta ophthalmologica 1980; 58: 507-511.

55. Becker R, Hubsch S, Graf MH, Kaufmann H. Examination of young children with Lea symbols. Br J Ophthalmol 2002; 86: 513-516.
56. Chandna A, Karki C, Davis J, Doran RML. Preferential looking in the mentally handicapped. Eye 1989; 3: 833-839.

57. Teller DY. The forced-choice preferential looking procedure: a psychophysical technique for use with human infants. Inf Behav and Dev 1979; 2: 135-153.

58. Teller DY, Mcdonald M, Preston K, Sebris SL, Dobson V. Assessment of visual acuity in infants and children: the acuity card procedure. Dev Med and Child Neurol 1986; 28: 779-789.

59. Marx MS, Werner P, Fridman P and Cohen-Mansfield J. Visual acuity estimates in the aged. Clin Vis Sci 4:179,1989.

60. Marx MS, Werner P, Cohen-Mansfield J, Hartmann EE. Visual acuity estimates in non-communicative elderly persons. Invest Ophthalmol Vis Sci 1990;31: 593596.

61. Friedman DS, Munoz B, Wassof RW et al. Grating visual acuity using the preferential-looking method in elderly nursing home residents. Invest Ophthalmol Vis Sci 2002; 43: 2572-2578.

62. Carabellese C, Appollonio I, Rozzini R, Bianchetti A, Frisoni GB, Frattola L, Trabucchi M. Sensory impairment and quality of life in a community elderly population. J Am Geriatr Soc 1993; 41:401-7.

62. Rovner BW, Zisselman PM, ShmuelyDulitzki Y. Depression and disability in older people with impaired vision: a follow-up study. J Am Geriatr Soc 1996; 44:181-184.

63. Chriqui E, Kergoat MJ, Champoux N, Leclerc BS, Kergoat H. Évaluation de l'acuité visuelle chez la personne âgée atteinte de troubles cognitifs. Association canadienne de gérontologie. Ottawa 2011. 